Internat. J. Math. \& Math. Sci.

Vol. 24, No. 9 (2000) 627-641

S0161171200004968

(C) Hindawi Publishing Corp.

\title{
ON COINCIDENCE AND COMMON FIXED POINTS OF NEARLY DENSIFYING MAPPINGS
}

\author{
ZEQING LIU and JEONG SHEOK UME
}

(Received 7 February 2000)

\begin{abstract}
Coincidence and common fixed point theorems for certain new classes of nearly densifying mappings are established. Our results extend, improve, and unify a lot of previously known theorems.
\end{abstract}

Keywords and phrases. Complete metric space, coincidence point, common fixed point, nearly densifying mapping, attractor, left reversible semigroup, F-diminishing orbital diameter.

2000 Mathematics Subject Classification. Primary 54H25.

1. Introduction. Furi and Vignoli [4] established first the existence of fixed point for densifying mappings. Afterwards Chatterjee [1], Diviccaro, Khan and Sessa [2], Fisher and Khan [3], Iseki [6, 7], Jain and Jain [8], Janos, Ko, and Tan [9], Khan [10], Khan and Fisher [11], Khan and Liu [12], Khan and Rao [13], Khan [14], Liu [17, 18, 19, 20, 21, 16], Pande [24, 25], Rao [22], Ray and Fisher [26], Sastry and Naidu [27], Sharma [28], Sharma and Srivastava [29] and others obtained fixed and coincidence point theorems for densifying and nearly densifying mappings, respectively. Huang, Huang, and Jeng [5] proved a common fixed point theorem for a left reversible semigroup, which consists of a number of continuous self-mappings in compact metric spaces.

The purpose of this paper is to establish coincidence and common fixed point theorems for certain new classes of nearly densifying mappings in complete metric spaces. In Section 2, we introduce notation, terminology and prove a lemma, which plays an important role in the paper. In Section 3, we obtain some common fixed point theorems for families of mappings. In Section 4, we give general coincidence point theorems for two pairs of mappings. Our results extend, improve, and unify the corresponding results of Chatterjee [1], Diviccaro, Khan, and Sessa [2], Huang, Huang, and Jeng [5], Janos, Ko, and Tan [9], Khan [10], Khan and Liu [12], Khan and Rao [13]. Liu $[17,18,19]$, Rao [22], Sharma and Srivastava [29] and others.

2. Preliminaries. Recall that a semigroup $G$ is said to be left reversible if for any $s, t \in G$ there exist $u, v \in G$ such that $s u=t v$. It is easy to see that the notion of left reversibility is equivalent to the statement that any two right ideals of $G$ have nonempty intersection. A semigroup $G$ is called near-commutative if for any $s, t \in$ $G$ there exists $u \in G$ such that $s t=t u$. Clearly, every commutative semigroup is near-commutative, and every near-commutative semigroup is left reversible, but the converses are not true. 
Throughout this paper, $(X, d)$ denotes a metric space, $\mathbb{N}, \mathbb{R}^{+}$, and $\mathbb{R}$ denote the sets of positive integers, nonnegative real numbers, and real numbers, respectively, and $\omega=\mathbb{N} \bigcup\{0\}$. Define

$$
\begin{aligned}
\mathfrak{I} & =\left\{F \mid F: X \times X \rightarrow \mathbb{R}^{+} \text {and } F(x, y)=0 \text { if and only if } x=y\right\}, \\
\mathfrak{I}_{1} & =\{F \mid F \in \mathfrak{I} \text { and } F \text { is upper semicontinuous in } X \times X\}, \\
\mathfrak{I}_{2} & =\{F \mid F \in \mathfrak{I} \text { and } F \text { is lower semicontinuous in } X \times X\} .
\end{aligned}
$$

Let $G$ be a family of self-mappings in $X$. A subset $Y$ of $X$ is called $G$-invariant if $g Y \subseteq Y$ for all $g \in G$. Let

$$
\begin{aligned}
N C I_{G} & =\{Y \mid Y \text { is nonempty compact } G \text {-invariant subset of } X\}, \\
C I S_{G} & =\left\{g \mid g: X \rightarrow X \text { and } g Y \subseteq Y, \forall Y \in N C I_{G}\right\},
\end{aligned}
$$

and $G^{*}$ be the semigroup generated by $G$ under composition. Clearly, $C I S_{G} \supseteq G^{*} \supseteq$ $\left\{g^{n}: n \in \omega\right\}$ for any $g \in G$. For $A, B \subseteq X, x, y \in X, f \in G$, and $F \in \mathfrak{T}$, define

$$
\begin{array}{ll}
\delta_{F}(A, B)=\sup \{F(a, b): a \in A, b \in B\}, & \delta_{F}(A)=\delta_{F}(A, A), \\
\delta_{F}(x, A)=\delta_{F}(\{x\}, A), & \delta_{F}(x, y)=\delta_{F}(\{x\},\{y\}), \\
O_{f}(x)=\left\{f^{n} x: n \in \omega\right\}, & O_{f}(x, y)=O_{f}(x) \bigcup O_{f}(y), \\
C_{f}=\{h \mid h: X \longrightarrow X, f h=h f\}, & G^{*} x=\{x\} \bigcup\left\{g x: g \in G^{*}\right\}, \\
C I S_{f}=C I S_{\{f\}}, & N C I_{f}=\operatorname{NCI}_{\{f\}} .
\end{array}
$$

$\bar{A}$ denotes the closure of $A$. $f$ is said to have diminishing orbital diameter if $\lim _{n \rightarrow \infty} \delta_{d}\left(O_{f}\left(f^{n} x\right)\right)<\delta_{d}\left(O_{f}(x)\right)$ for all $x \in X$ with $\delta_{d}\left(O_{f}(x)\right)>0 . \quad f$ is called contractive with respect to $d$ if $d(f x, f y)<d(x, y)$ for all distinct $x, y \in X$.

DEFINITION 2.1. Let $G$ be a semigroup of self-mappings on a metric space $(X, d)$ and $F \in \mathfrak{I} . G$ is said to have $F$-diminishing orbital diameter, if for any $x \in X$ with $\delta_{F}(G x)>0$ there is $s \in G$ such that $\delta_{F}(G s x)<\delta_{F}(G x)$.

DEFinITION 2.2 (see [15]). Let $A$ be a bounded subset of a metric space $(X, d)$. Then $\alpha(A)$, the measure of noncompactness of $A$, is the infimum of all $\varepsilon>0$ such that $A$ admits a finite covering consisting of subsets with diameters less than $\varepsilon$.

The following properties of $\alpha$ are well known.

LEMMA 2.3. Let $(X, d)$ be a metric space and $A, B$ be bounded subsets of $X$. Then

$$
\begin{gathered}
\alpha(A \cup B)=\max \{\alpha(A), \alpha(B)\} ; \\
\alpha(A)=0 \Longleftrightarrow A \text { is pre-compact, i.e., } A \text { is totally bounded; } \\
\alpha(A)=\alpha(\bar{A}) .
\end{gathered}
$$

Definition 2.4 (see [4]). A continuous self-mapping $f$ in a metric space $(X, d)$ is said to be densifying if $\alpha(f(A))<\alpha(A)$ for every bounded subset $A$ of $X$ with $\alpha(A)>0$. 
Definition 2.5 (see [27]). A self-mapping $f$ in a metric space $(X, d)$ is said to be nearly densifying if $\alpha(f(A))<\alpha(A)$ for every bounded and $f$-invariant subset $A$ of $X$ with $\alpha(A)>0$.

Obviously, each densifying mapping is nearly densifying, but the converse is false.

DefinITION 2.6 (see [23]). Let $X$ be a topological space, $f$ a self-mapping in $X$, and $M$ a nonempty subset of $X$. $M$ is an attractor for compact sets under $f$ if

(i) $M$ is compact and $f M \subseteq M$,

(ii) given any compact set $C \subseteq X$ and any open neighborhood $U$ of $M$, there exists $k \in N$ such that $f^{n} C \subseteq U$ for all $n \geq k$.

Let $G$ be a left reversible semigroup. We define a relation $\geq$ on $G$ by $a \geq b$ if and only if $a \in b G \bigcup\{b\}$. It is easy to check that $(G, \geq)$ is a directed set.

LEMмA 2.7. Let $G$ be a left reversible semigroup of continuous self-mappings in a compact metric space $(X, d), A=\bigcap_{f \in G} f x$, and $F \in \mathfrak{J}_{1}$. Then

$$
\begin{aligned}
& \lim _{f \in G} \delta_{F}(f x)=\delta_{F}(A) ; \\
& A \in N C I_{G} \quad \text { and } \quad f A=A, \quad \forall f \in G .
\end{aligned}
$$

Proof. Note that $f X \subseteq g X$ for all $f, g \in G$ with $f \geq g$. Thus $\left\{\delta_{F}(f X)\right\}_{f \in G}$ is a bounded decreasing net in $\mathbb{R}$. Obviously, $\lim _{f \in G} \delta_{f}(f X)$ exists in $\mathbb{R}$ and

$$
\delta_{F}(A) \leq \lim _{f \in G} \delta_{F}(f x) .
$$

We now prove that $f X$ is a compact subset of $X$ for each $f \in G$. Let $x$ be in $X$ and $\left\{x_{n}\right\}_{n \in \mathbb{N}} \subseteq X$ with $\lim _{n \rightarrow \infty} f x_{n}=x$. The compactness of $X$ ensures that there exists a subsequence $\left\{x_{n_{k}}\right\}_{k \in \mathbb{N}}$ of $\left\{x_{n}\right\}_{n \in \mathbb{N}}$ such that it converges to some point $t \in X$. Since $f$ is continuous, so $x=f t \in f X$. Therefore $f X$ is closed. That is, $f X$ is compact. This means that $A$ is compact.

We next prove that

$$
\delta_{F}(A) \geq \lim _{f \in G} \delta_{F}(f X) .
$$

Let $f \in G$. Since $F$ is upper semicontinuous and $f X \times f X$ is compact, there exist $x_{f}, y_{f} \in f X$ with $F\left(x_{f}, y_{f}\right)=\delta_{F}(f X)$. From the compactness of $X$ we can choose two subnets $\left\{x_{f_{k}}\right\}$ and $\left\{y_{f_{k}}\right\}$ of $\left\{x_{f}\right\}$ and $\left\{y_{f}\right\}$, respectively, such that $x_{f_{k}} \rightarrow x$ and $y_{f_{k}} \rightarrow y$ for some $x, y \in X$. For every $g \in G$ and $f_{k} \geq g$, we get that $x_{f_{k}}, y_{f_{k}} \in g X$. By virtue of closedness of $g X$, we infer that $x, y \in g X$. This means that $x, y \in A$. Consequently,

$$
\lim _{f \in G} \delta_{F}(f X)=\lim _{f \in G} F\left(x_{f}, y_{f}\right)=\lim _{k} F\left(x_{f_{k}}, y_{f_{k}}\right)=F(x, y) \leq \delta_{F}(A) .
$$

Thus (i) follows from (2.8) and (2.9).

Let $n \in \mathbb{N}$ and $f_{1}, f_{2}, \ldots, f_{n} \in G$. It follows from the left reversibility of $G$ that there exist $g_{1}, g_{2}, \ldots, g_{n} \in G$ with $f_{1} g_{1}=f_{2} g_{2}=\cdots=f_{n} g_{n}=h$ for some $h \in G$. Hence $\bigcap_{i=1}^{n} f_{i} X \supseteq h X \neq \varnothing$. The compactness of $X$ implies that $A \neq \varnothing$. 
We finally prove that $f A=A$ for all $f \in G$. Let $f \in G$ and $x \in A$. For any $g \in G$ there exist $a, b \in G$ with $f a=g b$. Note that $x \in A \subseteq a X$. Thus there is $y \in X$ with $x=a y$. It follows that $f x=f a y=g b y \in g X$. This implies that $f A \subseteq \bigcap_{g \in G} g X=A$ for $f \in G$. For the reverse inclusion, let $f, g \in G$ and $y \in A$. It follows from $y \in f g X$ that there exists $x_{g} \in g X$ with $f x_{g}=y$. The compactness $X$ ensures that there exists a convergent subnet $\left\{x_{g_{k}}\right\}$ of $\left\{x_{g}\right\}$ such that $x_{g_{k}} \rightarrow x$ for some $x \in X$. Therefore $y=f x$. For any $h, g \in G$ with $g \geq h$, we obtain that $h X$ is closed and $x_{g}$ belongs to $h X$. Thus the limit point $x$ of $\left\{x_{g}\right\}$ lies in $h X$. That is, $x \in A$. Note that $y=f x \in f A$. Therefore, $A \subseteq f A$ for $f \in G$. This completes the proof.

REMARK 2.8. Lemma 2.7 generalizes Lemma 2.3 of Huang, Huang, and Jeng [5].

\section{Common fixed point theorems for nearly densifying mappings}

THEOREM 3.1. Let $G$ and $H$ be finite families of continuous and nearly densifying self-mappings in a complete metric space $(X, d)$. If there exist $g \in G^{*}, h \in H^{*}, F \in$ $\mathfrak{I}_{1}, x_{0}, y_{0} \in X$ such that

$$
F(g x, h y)<\delta_{F}\left(\bigcup_{s \in C I S_{G}} s G^{*} x, \bigcup_{t \in C I S_{H}} t H^{*} y\right), \quad \forall x, y \in X \text { with } g x \neq h y ;
$$

$G^{*} x_{0}, H^{*} y_{0}$ are bounded and $G^{*}, H^{*}$ are left reversible.Then the following statements hold:

(i) $G$ and $H$ have a unique common fixed point $w \in X$, and $w$ is also the only fixed point of $G$ and $H$, respectively;

(ii) $\lim _{s \in G^{*}} F\left(s x_{0}, w\right)=\lim _{t \in H^{*}} F\left(t y_{0}, w\right)=\lim _{s \in G^{*}} \delta_{F}\left(s \overline{G^{*} x_{0}}\right)=\lim _{t \in H^{*}} \delta_{F}\left(t \overline{H^{*} y_{0}}\right)$ $=0$;

(iii) for any $C \in N C I_{G^{*}}$ and any $D \in N C I_{H^{*}}, \bigcap_{s \in G^{*}} S C=\bigcap_{t \in H^{*}} t D=\{w\}$.

Proof. Let $A=\bigcap_{s \in G^{*}} s \overline{G^{*} x_{0}}$ and $B=\bigcap_{t \in H^{*}} t \overline{H^{*} y_{0}}$. Since $G^{*} x_{0}=\left\{x_{0}\right\} \bigcup_{s \in G} s G^{*} x_{0}$ and $G$ is finite, so

$$
\alpha\left(G^{*} x_{0}\right)=\max \left\{\alpha\left(x_{0}\right), \alpha\left(s G^{*} x_{0}\right): s \in G\right\}=\max \left\{\alpha\left(s G^{*} x_{0}\right): s \in G\right\} .
$$

Note that each $s \in G$ is nearly densifying. Thus, $\alpha\left(G^{*} x_{0}\right)=0$. It follows from Lemma 2.3 that $G^{*} x_{0}$ is pre-compact. Completeness of $(X, d)$ ensures that $\overline{G^{*} x_{0}}$ is compact. Since every $s \in G^{*}$ is continuous, $s \overline{G^{*} x_{0}} \subseteq \overline{s G^{*} x_{0}} \subseteq \overline{G^{*} x_{0}}$. By Lemma 2.7 we immediately conclude that $A \in N C I_{G^{*}}$ and $f A=A$ for all $f \in G^{*}$. Similarly, $B \in N C I_{H^{*}}$ and $f B=B$ for all $f \in H^{*}$.

We assert that $\delta_{F}(A, B)=0$. Otherwise $\delta_{F}(A, B)>0$. Since $F$ is upper semicontinuous and $A \times B$ is compact, we can easily choose $a \in A$ and $b \in B$ with $F(a, b)=\delta_{F}(A, B)$. Therefore, there exist $x \in A$ and $y \in B$ such that $a=g x$ and $b=h y$. Using (3.1), we have

$$
\begin{aligned}
F(a, b) & =F(g x, h y)<\delta_{F}\left(\bigcup_{s \in C I S_{G}} s G^{*} x, \bigcup_{t \in C I S_{H}} t H^{*} y\right) \\
& \leq \delta_{F}(A, B)=F(a, b),
\end{aligned}
$$


which is a contradiction. Consequently, $A=B=a$ singleton, say, $\{w\}$ for some $w \in X$. Thus $w=f w$ for all $f \in G \cup H$. That is, $G$ and $H$ have a common fixed point $w \in X$. If $G$ has another fixed point $v \in X$ and $v \neq w$, by (3.1) we infer that

$$
F(v, w)=F(g v, h w)<\delta_{F}\left(\bigcup_{s \in C I S_{G}} s G^{*} v, \bigcup_{t \in C I S_{H}} t H^{*} w\right)=F(v, w),
$$

which is absurd. Hence $G$ has a unique fixed point $w$. Similarly, we conclude that $H$ has also a unique fixed point $w$.

It follows from Lemma 2.7 that

$$
\lim _{s \in G^{*}} \delta_{F}\left(s \overline{G^{*} x_{0}}\right)=\delta_{F}(A)=\delta_{F}(\{w\})=0=\delta_{F}(B)=\lim _{t \in H^{*}} \delta_{F}\left(t \overline{H^{*} y_{0}}\right) .
$$

Note that $s x_{0} \in s G^{*} x_{0}, t y_{0} \in t H^{*} y_{0}$ and $w \in \overline{s G^{*} x_{0}} \cap t \overline{H^{*} y_{0}}$ for all $s \in G^{*}, t \in H^{*}$. Thus Theorem 3.1(ii) follows immediately from (3.5).

Let $C \in N C I_{G^{*}}$ and $Y=\bigcap_{s \in G^{*}} S C$. Lemma 2.7 ensures that $Y \in N C I_{G^{*}}$ and $f Y=Y$ for all $f \in G^{*}$. Suppose that $\delta_{F}(Y, w)>0$. Then there exists $x \in Y$ such that $F(g x, w)=\delta_{F}(Y, w)$. In view of (3.1) and Theorem 3.1(i). We obtain that $F(g x, w)<$ $\delta_{F}\left(\bigcup_{s \in C I S_{G}} s G^{*} x, w\right) \leq \delta_{F}(Y, w)$, which is impossible. Hence $\delta_{F}(y, w)=0$. That is, $Y=\bigcap_{s \in G^{*}} S C=\{w\}$. Similarly, we obtain that $\bigcap_{t \in H^{*}} t D=\{w\}$ if $D \in N C I_{H^{*}}$. This completes the proof.

THEOREM 3.2. Let $G$ and $H$ be finite families of continuous and nearly densifying self-mappings in a complete bounded metric space $(X, d)$ satisfying (3.1). Assume that $G^{*}, H^{*}$ are near commutative. Then Theorem 3.1(i), (iii), and the following statements hold:

(i)

$$
\begin{aligned}
\lim _{s \in G^{*}} F(s x, w) & =\lim _{t \in H^{*}} F(t y, w)=\lim _{s \in G^{*}} \delta_{F}\left(s \overline{G^{*} x}\right) \\
& =\lim _{t \in H^{*}} \delta_{F}\left(t \overline{H^{*} y}\right)=0, \quad \forall x, y \in X ;
\end{aligned}
$$

(ii) $G^{*}$ and $H^{*}$ have F-diminishing orbital diameter.

Proof. Let $x, y$ be in $X$. Put $A=\bigcap_{s \in G^{*}} s \overline{G^{*} x}$ and $B=\bigcap_{t \in H^{*}} t \overline{H^{*} y}$. As in the proof of Theorem 3.1, we conclude that $A \in N C I_{G^{*}}, f A=A$ for all $f \in G^{*}$ and $B \in N C I_{H^{*}}$, $g B=B$ for all $g \in H^{*}$. It follows from Theorem 3.1(ii) that

$$
A=\bigcap_{s \in G^{*}} s A=\{w\}=B=\bigcap_{t \in H^{*}} t B .
$$

Thus (3.6) follows from Lemma 2.7 and the definitions of $G^{*} x$ and $H^{*} y$.

Given $s, t \in G^{*}$. Since $G^{*}$ is commutative, there is $g \in G^{*}$ with $t s=s g$. This means that

$$
\delta_{F}\left(G^{*} s x\right)=\delta_{F}\left(\{s x\} \bigcup\left\{t s x: t \in G^{*}\right\}\right) \leq \delta_{F}\left(s G^{*} x\right) \leq \delta_{F}\left(s \overline{G^{*} x}\right) .
$$

Suppose that $\delta_{F}\left(G^{*} s x\right)>0$. In view of (3.6) and (3.8) there exists $s \in G^{*}$ such that $\delta_{F}\left(G^{*} s x\right)<\delta_{F}\left(G^{*} x\right)$. That is, $G^{*}$ has $F$-diminishing orbital diameter. Analogously, $H^{*}$ has $F$-diminishing orbital diameter also. This completes the proof. 
We now state without proof analogues of Theorems 3.1 and 3.2.

THEOREM 3.3. Let $G$ be a finite family of continuous and nearly densifying selfmappings in a complete metric space $(X, d)$. If there exist $g, h \in G^{*}, F \in \mathfrak{I}_{1}, x_{0} \in X$ such that

$$
F(g x, h y)<\delta_{F}\left(\bigcup_{s \in C I S_{G}}\left(s G^{*} x \bigcup s G^{*} y\right)\right), \quad \forall x, y \in X \text { with } g x \neq h y
$$

$G^{*} x_{0}$ is bounded and $G^{*}$ is left reversible.Then the following statements hold:

(i) $G$ has a unique common fixed point $w \in X$, and

$$
\lim _{s \in G^{*}} F\left(s x_{0}, w\right)=\lim _{s \in G^{*}} \delta_{F}\left(s \overline{G^{*} x_{0}}\right)=0
$$

(ii) for any $C \in N C I_{G^{*}}, \bigcap_{s \in G^{*}} S C=\{w\}$.

THEOREM 3.4. Let $f$ and $g$ be continuous self-mappings in a complete metric space $(X, d)$. Assume that there exist $i, j, p, q \in \mathbb{N}, F \in \mathfrak{I}_{1}, x_{0}, y_{0} \in X$ such that

(i) $F\left(f^{p} x, g^{q} y\right)<\delta_{F}\left(\bigcup_{s \in C I S_{f}} s O_{f}(x), \bigcup_{t \in C I S_{g}} t O_{g}(y)\right), \forall x, y \in X$ with $f^{p} x \neq g^{q} y$;

(ii) $f^{i}$ and $g^{j}$ are nearly densifying;

(iii) $O_{f}\left(x_{0}\right)$ and $O_{g}\left(y_{0}\right)$ are bounded.

Then the following statements hold:

(1) $f$ and $g$ have a unique common fixed point $w \in X$, and $w$ is also the only fixed point of $f$ and $g$, respectively;

(2) $\lim _{n \rightarrow \infty} F\left(f^{n} x_{0}, w\right)=\lim _{n \rightarrow \infty} F\left(g^{n} y_{0}, w\right)=\lim _{n \rightarrow \infty} \delta_{F}\left(f^{n} \overline{O_{f}\left(x_{0}\right)}\right)=$ $\lim _{n \rightarrow \infty} \delta_{F}\left(g^{n} \overline{O_{g}\left(y_{0}\right)}\right)=0$;

(3) for any $C \in N C I_{f}$ and any $D \in N C I_{g}$,

$$
\bigcap_{n \in \mathbb{N}} f^{n} C=\bigcap_{n \in \mathbb{N}} g^{n} D=\{w\}
$$

Proof. Set $A=\bigcap_{n \in \mathbb{N}} f^{n} \overline{O_{f}\left(x_{0}\right)}$ and $B=\bigcap_{n \in \mathbb{N}} g^{n} \overline{O_{g}\left(y_{0}\right)}$. In view of Theorem 3.4(ii), (iii) and

$$
\alpha\left(O_{f}\left(x_{0}\right)\right)=\max \left\{\alpha\left\{f^{k} x_{0}: 0 \leq k \leq i-1\right\}, \alpha\left(f^{i} O_{f}\left(x_{0}\right)\right)\right\},
$$

we conclude easily that $A \in N C I_{f}$ and $f A=A$. Similarly, $B \in N C I_{g}$ and $g B=B$. The rest of the proof is the same as that of Theorem 3.1. This completes the proof.

REMARK 3.5. Theorem 3.4 extends Theorems 3 and 4 of Liu [19], the theorem of Sharma and Srivastava [29]. Akin to Theorem 3.4, we have the following.

THEOREM 3.6. Let $f$ be continuous self-mapping in a complete metric space $(X, d)$. Assume that there exist $i, p, q \in \mathbb{N}, F \in \mathfrak{I}_{1}, x_{0} \in X$ such that

(i) $F\left(f^{p} x, f^{q} y\right)<\delta_{F}\left(\bigcup_{s \in C I S_{f}} s O_{f}(x, y)\right), \forall x, y \in X$ with $f^{p} x \neq f^{q} y$;

(ii) $f^{i}$ is nearly densifying;

(iii) $O_{f}\left(x_{0}\right)$ is bounded. 
Then the following statements hold:

(1) $f$ has a unique fixed point $w \in X$, and $\lim _{n \rightarrow \infty} F\left(f^{n} x_{0}, w\right)=\lim _{n \rightarrow \infty} \delta_{F}\left(f^{n} \overline{O_{f}\left(x_{0}\right)}\right)$ $=0$;

(2) for any $C \in N C I_{f}, \bigcap_{n \in \mathbb{N}} f^{n} C=\{w\}$.

REMARK 3.7. Theorem 4 of Khan [10] and Theorem 4 of Rao [22] are special cases of Theorem 3.6.

THEOREM 3.8. Let $f$ and $g$ be continuous self-mappings in a complete bounded metric space $(X, d)$. Assume that there exist $i, j, p, q \in \mathbb{N}$ satisfying Theorem 3.4(ii) and

$$
\begin{array}{r}
d\left(f^{p} x, g^{q} y\right)<\delta_{d}\left(\bigcup_{s \in C I S_{f}} s O_{f}(x), \bigcup_{t \in C I S_{g}} t O_{g}(y)\right), \\
\forall x, y \in X \text { with } f^{p} x \neq g^{q} y .
\end{array}
$$

Then Theorem 3.4(1) and (3.11) and the following statements hold:

(i) $\lim _{n \rightarrow \infty} d\left(f^{n} x, w\right)=\lim _{n \rightarrow \infty} d\left(g^{n} y, w\right)=\lim _{n \rightarrow \infty} \delta_{d}\left(f^{n} O_{f}(x)\right)=$ $\lim _{n \rightarrow \infty} \delta_{d}\left(g^{n} O_{g}(y)\right)=0, \forall x, y \in X$;

(ii) there exist bounded complete metrics $d_{1}, d_{2}$ on $X$ which are equivalent to $d$ such that $f, g$ are contractive with respect to $d_{1}$ and $d_{2}$, respectively;

(iii) $C I S_{f}$ and $C I S_{g}$ have a unique common fixed point $w \in X$, and $w$ is also the only fixed point of CIS $f$ and $C I S_{g}$, respectively;

(iv) $f$ and $g$ have diminishing orbital diameter.

Proof. It follows from Theorem 3.4 that Theorem 3.4(1), (3.11), and Theorem 3.8(i) hold. By the definitions of $C I S_{f}$ and $C I S_{g}$, we conclude easily that Theorem 3.8(iii) holds. Since $f^{n} O_{f}(x)=O_{f}\left(f^{n} x\right)$ and $g^{n} O_{g}(y)=O_{g}\left(g^{n} y\right)$, so Theorem 3.6(iv) is satisfied. Now we prove that Theorem 3.8(ii) holds. Assume that $B$ be any nonempty compact subset of $X$. Using Lemma 2.3, we have

$$
\begin{aligned}
\alpha\left(\bigcup_{n \in \omega} f^{n} B\right) & =\max \left\{\alpha\left(\bigcup_{n=0}^{i-1} f^{n} B\right), \alpha\left(\bigcup_{n=i}^{\infty} f^{n} B\right)\right\} \\
& =\alpha\left(\bigcup_{n=i}^{\infty} f^{n} B\right)=\alpha\left(f^{i} \bigcup_{n \in \omega} f^{n} B\right) .
\end{aligned}
$$

Thus $\bigcup_{n \in \omega} f^{n} B$ is totally bounded because $f^{i}$ is nearly densifying. Set $C=\overline{\bigcup_{n \in \omega} f^{n} B}$. Since $f$ is continuous and $X$ is complete, we infer that $C$ is compact and $f C \subseteq$ $\overline{f \bigcup_{n \in \omega} f^{n} B} \subseteq C$. Hence (3.11) ensures that $\bigcap_{n \in \omega} f^{n} C=\{w\}$. This means that $\delta_{d}\left(f^{n} C\right) \downarrow 0$ as $n \rightarrow \infty$. For each open neighborhood $U$ of $w$, there exists an open ball $B(w, \varepsilon)=\{x: x \in X$ and $d(x, w)<\varepsilon\}$ with $B(w, \varepsilon) \subseteq U$. Note that $\delta_{d}\left(f^{n} C\right) \downarrow 0$ as $n \rightarrow \infty$. Thus there exists $k \in \mathbb{N}$ such that $\delta_{d}\left(f^{n} C\right)<\varepsilon$ for all $n \geq k$. Given $x \in f^{n} C$ and $n \geq k$, we obtain that $d(x, w) \leq \delta_{d}\left(f^{n} C\right)<\varepsilon$. Consequently, $f^{n} B \subseteq f^{n} C \subseteq$ $B(w, \varepsilon) \subseteq U$ for all $n \geq k$. This shows that $\{w\}$ is an attractor for compact sets under $f$. Thus Theorem 3.8(ii) follows from theorem of [9] and Remark 1 of [9]. This completes the proof.

Similarly, we have the following theorem. 
THEOREM 3.9. Let $f$ be a continuous self-mapping in a complete bounded metric space $(X, d)$. Assume that there exist $i, p, q \in \mathbb{N}$ satisfying Theorem 3.6(ii) and

$$
d\left(f^{p} x, f^{q} y\right)<\delta_{d}\left(\bigcup_{s \in C I S_{f}} s O_{f}(x, y)\right), \quad \forall x, y \in X \text { with } f^{p} x \neq f^{q} y
$$

Then Theorem 3.6(2) and the following statements hold:

(i) $f$ has a unique fixed point $w \in X$, and has diminishing orbital diameter and $\lim _{n \rightarrow \infty} d\left(f^{n} x, w\right)=\lim _{n \rightarrow \infty} \delta_{d}\left(f^{n} O_{f}(x)\right)=0, \forall x \in X$

(ii) there exists a bounded complete metric $d_{1}$ on $X$ which is equivalent to $d$ such that $f$ is contractive with respect to $d_{1}$;

(iii) $C I S_{f}$ has a unique common fixed point $w \in X$.

REMARK 3.10. Theorem 3.8 generalizes Theorem 4 of [2] and Theorem 4 of [22]. Theorem 3.9 extends and improves Theorem 3 of [1], Corollary 2 of [9], Theorem 3.1 of [17], and Theorems 1 and 2 of [18]

\section{Coincidence point theorems for two pairs of nearly densifying mappings}

THEOREM 4.1. Let $f, g, s$, and $t$ be a continuous and nearly densifying mappings from a complete metric space $(X, d)$ into itself satisfying

$$
f g t=f t g=t f g \quad \text { and } \quad g s t=s g t=s t g .
$$

Let $G=\{f, g, s, t\}$. Assume that there exist $F_{1}, F_{2} \in \mathfrak{I}$ and $x_{0} \in X$ such that

$$
\begin{aligned}
& F_{1} \text { or } F_{2} \in \mathfrak{I}_{2} \text {; } \\
& F_{1}(f x, g y)<\max \left\{F_{2}(s x, t y), F_{2}(s x, f x), F_{1}(t y, g y),\right. \\
& \min \left\{F_{2}(s x, g y), F_{1}(f x, t y)\right\}, \frac{\left[F_{2}(s x, t y)\right]^{2}}{F_{1}(f x, g y)}, \\
& \frac{\left[F_{2}(s x, f x)\right]^{2}}{F_{1}(f x, g y)}, \frac{\left[F_{1}(t y, g y)\right]^{2}}{F_{1}(f x, g y)}, \\
& \frac{F_{2}(s x, t y) F_{1}(f x, t y)}{F_{1}(f x, g y)}, \frac{F_{2}(s x, f x) F_{1}(f x, t y)}{F_{1}(f x, g y)}, \\
& \frac{F_{1}(t y, g y) F_{1}(f x, t y)}{F_{1}(f x, g y)}, \frac{F_{2}(s x, g y) F_{1}(f x, t y)}{F_{1}(f x, g y)}, \\
& \frac{\left[F_{2}(s x, f x)\right]^{2}}{F_{2}(s x, t y)}, \frac{F_{2}(s x, f x) F_{1}(t y, g y)}{F_{2}(s x, t y)}, \\
& \frac{F_{2}(s x, f x) F_{1}(f x, g y)}{F_{2}(s x, t y)}, \frac{F_{2}(s x, f x) F_{1}(f x, t y)}{F_{2}(s x, t y)}, \\
& \left.\frac{F_{1}(t y, g y) F_{1}(f x, t y)}{F_{2}(s x, t y)}, \frac{F_{2}(s x, g y) F_{1}(s x, t y)}{F_{2}(s x, t y)}\right\}
\end{aligned}
$$


for all $x, y \in X$ with $s x \neq t y, f x \neq g y$;

$$
\begin{aligned}
F_{2}(g x, f y)<\max \{ & F_{1}(t x, s y), F_{1}(t x, g x), F_{2}(s y, f y), \\
& \min \left\{F_{1}(g x, s y), F_{2}(t x, f y)\right\}, \frac{\left[F_{1}(t x, s y)\right]^{2}}{F_{2}(g x, f y)}, \\
& \frac{\left[F_{1}(t x, g x)\right]^{2}}{F_{2}(g x, f y)}, \frac{\left[F_{2}(s y, f y)\right]^{2}}{F_{2}(g x, f y)}, \frac{F_{1}(t x, s y) F_{1}(g x, s y)}{F_{2}(g x, f y)}, \\
& \frac{F_{1}(t x, g x) F_{1}(g x, s y)}{F_{2}(g x, f y)}, \frac{F_{2}(s y, f y) F_{1}(g x, s y)}{F_{2}(g x, f y)}, \\
& \frac{F_{2}(t x, f y) F_{1}(g x, s y)}{F_{2}(g x, f y)}, \frac{\left[F_{1}(t x, g x)\right]^{2}}{F_{1}(t x, s y)}, \frac{F_{1}(t x, g x) F_{2}(s y, f y)}{F_{1}(t x, s y)}, \\
& \frac{F_{1}(t x, g x) F_{2}(g x, f y)}{F_{1}(t x, s y)}, \frac{F_{1}(t x, g x) F_{1}(g x, s y)}{F_{2}(t x, s y)}, \\
& \left.\frac{F_{2}(s y, f y) F_{1}(g x, s y)}{F_{1}(t x, s y)}, \frac{F_{1}(g x, s y) F_{2}(t x, f y)}{F_{1}(t x, s y)}\right\}
\end{aligned}
$$

for all $x, y \in X$ with $g x \neq f y, t x \neq s y$;

$$
G^{*} x_{0} \text { is bounded and } G^{*} \text { is left reversible. }
$$

Then $f$ and $s$ or $g$ and $t$ have a coincidence point in $X$.

Proof. Put $A=G^{*} x_{0}$. It follows that $A=\left\{x_{0}\right\} \cup f A \cup g A \cup s A \cup t A$. This yields that

$$
\alpha(A)=\max \{\alpha(f A), \alpha(g A), \alpha(s A), \alpha(t A)\} .
$$

It is evident to see that $\alpha(A)=0$. Thus $\bar{A}$ is compact by completeness of $X$. Set $B \bigcap_{h \in G^{*}} h \bar{A}$. Lemma 2.7 ensures that $f B=g B=s B=t B=B \neq \varnothing$ and $B$ is compact. Let $F_{1}$ be in $\mathfrak{J}_{2}$. Define $r: B \rightarrow \mathbb{R}^{+}$by putting $r(x)=F_{1}(t x, g x)$. Since $r$ is a lower semi-continuous function on the compact set $B$, so there exists $b \in B$ with

$$
r(b)=F_{1}(t b, g b)=\inf _{x \in B} F_{1}(t x, g x) .
$$

Suppose that neither $f$ and $s$ nor $g$ and $t$ have a coincidence point. Then

$$
t f g c \neq g f g c, \quad t s t c \neq g s t c, \quad s t g c \neq f t g c,
$$

where $b=s t c \in B$. In view of (4.1), (4.3), (4.4), (4.7) and (4.8), we have

$$
\begin{aligned}
r(f g c)= & F_{1}(t f g c, g f g c)=F_{1}(f t g c, g f g c) \\
<\max \{ & F_{2}(\operatorname{stgc}, t f g c), F_{2}(s t g c, f t g c), F_{1}(t f g c, g f g c), \\
& \min \left\{F_{2}(s t g c, g f g c), F_{1}(f t g c, t f g c)\right\} \frac{\left[F_{2}(s t g c, t f g c)\right]^{2}}{F_{1}(f t g c, g f g c)}, \\
& \frac{\left[F_{2}(\operatorname{stgc}, f t g c)\right]^{2}}{F_{1}(f t g c, g f g c)}, \frac{\left[F_{1}(t f g c, g f g c)\right]^{2}}{F_{1}(f t g c, g f g c)},
\end{aligned}
$$




$$
\begin{aligned}
& \frac{F_{2}(\operatorname{stg} c, t f g c) F_{1}(f t g c, t f g c)}{F_{1}(f t g c, g f g c)}, \frac{F_{2}(s t g c, f t g c) F_{1}(f t g c, t f g c)}{F_{1}(f t g c, g f g c)}, \\
& \frac{F_{1}(t f g c, g f g c) F_{1}(f t g c, t f g c)}{F_{1}(f t g c, g f g c)}, \frac{F_{2}(s t g c, g f g c) F_{1}(f t g c, t f g c)}{F_{1}(f t g c, g f g c)}, \\
& \frac{\left[F_{2}(\operatorname{stgc}, f t g c)\right]^{2}}{F_{2}(\operatorname{stgc}, t f g c)}, \frac{F_{2}(s t g c, f t g c) F_{1}(t f g c, g f g c)}{F_{2}(s t g c, t f g c)}, \\
& \frac{F_{2}(s t g c, f t g c) F_{1}(f t g c, g f g c)}{F_{2}(s t g c, t f g c)}, \frac{F_{2}(s t g c, f t g c) F_{1}(f t g c, t f g c)}{F_{2}(s t g c, t f g c)}, \\
& \left.\frac{F_{1}(t f g c, g f g c) F_{1}(f t g c, t f g c)}{F_{2}(s t g c, t f g c)}, \frac{F_{2}(s t g c, g f g c) F_{1}(f t g c, t f g c)}{F_{2}(s t g c, t f g c)}\right\} \\
& =\max \left\{F_{2}(g s t c, f g t c), F_{2}(g s t c, f g t c), r(f g c), 0, \frac{\left[F_{2}(g s t c, f g t c)\right]^{2}}{r(f g c)},\right. \\
& \frac{\left[F_{2}(g s t c, f g t c)\right]^{2}}{r(f g c)}, r(f g c), 0,0,0,0, F_{2}(g s t c, f g t c), \\
& r(f g c), r(f g c), 0,0,0\} \\
& =\max \left\{F_{2}(g s t c, f g t c), \frac{\left[F_{2}(g s t c, f g t c)\right]^{2}}{r(f g c)}\right\} \\
& =F_{2}(g s t c, f g t c) \\
& <\max \left\{F_{1}(t s t c, s g t c), F_{1}(t s t c, g s t c), F_{2}(s g t c, f g t c)\right. \\
& \min \left\{F_{1}(g s t c, s g t c), F_{2}(t s t c, f g t c)\right\}, \frac{\left[F_{1}(t s t c, s g t c)\right]^{2}}{F_{2}(g s t c, f g t c)}, \\
& \frac{\left[F_{1}(t s t c, g s t c)\right]^{2}}{F_{2}(g s t c, f g t c)}, \frac{\left[F_{2}(s g t c, f g t c)\right]^{2}}{F_{2}(\text { gstc }, f g t c)}, \\
& \frac{F_{1}(t s t c, s g t c) F_{1}(g s t c, s g t c)}{F_{2}(g s t c, f g t c)}, \frac{F_{1}(t s t c, g s t c) F_{1}(g s t c, s g t c)}{F_{2}(g s t c, f g t c)}, \\
& \frac{F_{2}(s g t c, f g t c) F_{1}(g s t c, s g t c)}{F_{2}(g s t c, f g t c)}, \frac{F_{2}(t s t c, f g t c) F_{1}(g s t c, s g t c)}{F_{2}(g s t c, f g t c)}, \\
& \frac{\left[F_{1}(t s t c, g s t c)\right]^{2}}{F_{1}(t s t c, s g t c)}, \frac{F_{1}(t s t c, g s t c) F_{2}(s g t c, f g t c)}{F_{1}(t s t c, s g t c)}, \\
& \frac{F_{1}(t s t c, g s t c) F_{2}(g s t c, f g t c)}{F_{1}(t s t c, s g t c)}, \frac{F_{1}(t s t c, g s t c) F_{1}(g s t c, s g t c)}{F_{1}(t s t c, s g t c)}, \\
& \left.\frac{F_{2}(s g t c, f g t c) F_{1}(g s t c, s g t c)}{F_{1}(t s t c, s g t c)}, \frac{F_{1}(g s t c, s g t c) F_{2}(t s t c, f g t c)}{F_{1}(t s t c, s g t c)}\right\} \\
& =\max \left\{r(b), r(b), F_{2}(g s t c, f g t c), 0, \frac{[r(b)]^{2}}{F_{2}(g s t c, f g t c)}, \frac{[r(b)]^{2}}{F_{2}(g s t c, f g t c)},\right. \\
& F_{2}(\text { gstc }, f g t c), 0,0,0,0, r(b), \\
& \left.F_{2}(g s t c, f g t c), F_{2}(g s t c, f g t c), 0,0,0\right\} \\
& =r(b) \text {, }
\end{aligned}
$$


which implies that

$$
r(b) \leq r(f g c)<r(b)
$$

which is a contradiction. Hence $f$ and $s$ or $g$ and $t$ must have a coincidence point. The argument is similar for $F_{2} \in \mathfrak{I}_{2}$. This completes the proof.

THEOREM 4.2. Let $f, g, s$, and $t$ be continuous and nearly densifying mappings from a complete metric space $(X, d)$ into itself satisfying $f, g \in C_{s} \cap C_{t}$. Let $G=$ $\{f, g, s, t\}$ and $H=\{s, t\}$. Assume that there exist $F_{1}, F_{2} \in \mathfrak{I}$ and $x_{0} \in X$ such that (4.2), (4.3), (4.4), and the following statement hold:

$$
G^{*} x_{0} \text { is bounded and } H^{*} \text { is left reversible. }
$$

Then $f$ and $s$ or $g$ and $t$ have a coincidence point in $X$.

Proof. Put $A=G^{*} x_{0}$ and $B=\bigcap_{h \in H^{*}} h \bar{A}$. As in the proof of Theorem 4.1, we infer that $B$ is nonempty compact subset of $\bar{A}$ and $s B=t B=B \supseteq f B \cup g B$. The remaining part of the proof is as in Theorem 4.1. This completes the proof.

REMARK 4.3. Theorem 3.1 of [12] and Theorem 3.1 of [13] are special cases of Theorem 4.2.

REMARK 4.4. The following example reveals that $f, g, s$, and $t$ in Theorems 4.1 and 4.2 do not necessarily have a coincidence point and that if either $f$ and $s$ or $g$ and $t$ have a coincidence point, then the coincidence point may not be unique.

EXAMPLE 4.5. Let $X=\{1,3,6\}$ with the usual metric $d$ and $F_{1}=F_{2}=d$. Define $f, g, s, t: X \rightarrow X$ by $f 1=g 3=g 6=1, f 3=f 6=g 1=3$ and $s=t=i_{X}$-the identity mapping on $X$. Take $G=\{f, g, s, t\}$ and $H=\{s, t\}$. Clearly, $g^{2}=f=f^{2}, g=f g=$ $g f=g^{3}, G=G^{*}, H=H^{*}$, and $G^{*}$ and $H^{*}$ are left reversible. It is easy to verify that

$$
d(f x, g y)=2<3=d(s x, t y)
$$

for all $x, y \in X$ with $s x \neq t y, f x \neq g y$, and

$$
d(g x, f y)=2<3=d(t x, s y)
$$

for all $x, y \in X$ with $t x \neq s y, g x \neq f y$. Thus the conditions of Theorems 4.1 and 4.2 are satisfied. However, $f$ and $s$ have two coincidence points 1 and 3 , while $f, g, s$, and $t$ have none.

THEOREM 4.6. Let $f, g, s$, and $t$ be continuous and nearly densifying mappings from a complete metric space $(X, d)$ into itself satisfying $f, g, s \in C_{t}$ and $g \in C_{s}$. Assume that there exist $F_{1}, F_{2} \in \mathfrak{I}$ and $x_{0} \in X$ satisfying (4.2), (4.3), and (4.4). If $b$ is a common coincidence point of $f, g, s$, and $t$, then $t b$ is a unique common fixed point of $f, g, s$, and $t$. 
Proof. Since $f, g, s \in C_{t}, g \in C_{s}$, and $f b=g b=s b=t b$, we have $t^{2} b=t f b=$ $f t b=t g b=g t b=t s b=s t b$. Suppose that $t^{2} b \neq t b$. From (4.3) and (4.4) we conclude that

$$
\begin{aligned}
& F_{1}\left(t^{2} b, t b\right)=F_{1}(f t b, g b) \\
& <\max \left\{F_{2}(s t b, t b), F_{2}(s t b, f t b), F_{1}(t b, g b),\right. \\
& \min \left\{F_{2}(s t b, g b), F_{1}(f t b, t b)\right\}, \frac{\left[F_{2}(s t b, t b)\right]^{2}}{F_{1}(f t b, g b)}, \\
& \frac{\left[F_{2}(s t b, f t b)\right]^{2}}{F_{1}(f t b, g b)}, \frac{\left[F_{1}(t b, g b)\right]^{2}}{F_{1}(f t b, g b)}, \frac{F_{2}(s t b, t b) F_{1}(f t b, t b)}{F_{1}(f t b, g b)}, \\
& \frac{F_{2}(s t b, f t b) F_{1}(f t b, t b)}{F_{1}(f t b, g b)}, \frac{F_{1}(t b, g b) F_{1}(f t b, t b)}{F_{1}(f t b, g b)}, \\
& \frac{F_{2}(s t b, g b) F_{1}(f t b, t b)}{F_{1}(f t b, g b)}, \frac{\left[F_{2}(s t b, f t b)\right]^{2}}{F_{2}(s t b, t b)}, \frac{F_{2}(s t b, f t b) F_{1}(t b, g b)}{F_{2}(s t b, t b)}, \\
& \frac{F_{2}(s t b, f t b) F_{1}(f t b, g b)}{F_{2}(s t b, t b)}, \frac{F_{2}(s t b, f t b) F_{1}(f t b, g b)}{F_{2}(s t b, t b)}, \\
& \left.\frac{F_{1}(t b, g b) F_{1}(f t b, t b)}{F_{2}(s t b, t b)}, \frac{F_{2}(s t b, g b) F_{1}(f t b, t b)}{F_{2}(s t b, t b)}\right\} \\
& =\max \left\{F_{2}\left(t^{2} b, t b\right), \frac{\left[F_{2}\left(t^{2} b, t b\right)\right]^{2}}{F_{1}\left(t^{2} b, t b\right)}, F_{1}\left(t^{2} b, t b\right)\right\} \\
& =F_{2}\left(t^{2} b, t b\right)=F_{2}(g t b, f b) \\
& <\max \left\{F_{1}\left(t^{2} b, s b\right), F_{1}\left(t^{2} b, g t b\right), F_{2}(s b, f b),\right. \\
& \min \left\{F_{1}(g t b, s b), F_{2}\left(t^{2} b, f b\right)\right\}, \frac{\left[F_{1}\left(t^{2} b, s b\right)\right]^{2}}{F_{2}(g t b, f b)}, \\
& \frac{\left[F_{1}\left(t^{2} b, g t b\right)\right]^{2}}{F_{2}(g t b, f b)}, \frac{\left[F_{2}(s b, f b)\right]^{2}}{F_{2}(g t b, f b)}, \frac{F_{1}\left(t^{2} b, s b\right) F_{1}(g t b, s b)}{F_{2}(g t b, f b)}, \\
& \frac{F_{1}\left(t^{2} b, g t b\right) F_{1}(g t b, s b)}{F_{2}(g t b, f b)}, \frac{F_{2}(s b, f b) F_{1}(g t b, s b)}{F_{2}(g t b, f b)}, \\
& \frac{F_{2}\left(t^{2} b, f b\right) F_{1}(g t b, s b)}{F_{2}(g t b, f b)}, \frac{\left[F_{1}\left(t^{2} b, g t b\right)\right]^{2}}{F_{1}\left(t^{2} b, s b\right)}, \\
& \frac{F_{1}\left(t^{2} b, g t b\right) F_{2}(s b, f b)}{F_{1}\left(t^{2} b, s b\right)}, \frac{F_{1}\left(t^{2} b, g t b\right) F_{2}(g t b, f b)}{F_{1}\left(t^{2} b, s b\right)}, \\
& \frac{F_{1}\left(t^{2} b, g t b\right) F_{1}(g t b, s b)}{F_{1}\left(t^{2} b, s b\right)}, \frac{F_{2}(s b, f b) F_{1}(g t b, s b)}{F_{1}\left(t^{2} b, s b\right)}, \\
& \left.\frac{F_{1}(g t b, s b) F_{2}\left(t^{2} b, f b\right)}{F_{1}\left(t^{2} b, s b\right)}\right\} \\
& =\max \left\{F_{1}\left(t^{2} b, t b\right), \frac{\left[F_{1}\left(t^{2} b, t b\right)\right]^{2}}{F_{2}\left(t^{2} b, t b\right)}, F_{2}\left(t^{2} b, t b\right)\right\} \\
& =F_{1}\left(t^{2} b, t b\right) \text {, }
\end{aligned}
$$

which is a contradiction. Therefore $t b=t^{2} b=f t b=g t b=s t b$. That is, $t b$ is a 
common fixed point of $f, g, s$, and $t$. The uniqueness of a common fixed point follows from (4.3) and (4.4). This completes the proof.

REMARK 4.7. Theorem 4.6 extends Theorem 3.2 of [12] and Theorem 3.2 of [13].

THEOREM 4.8. Let $f, g, s$, and $t$ be continuous and nearly densifying mappings from a complete metric space $(X, d)$ into itself and $G=\{f, g, s, t\}$. Suppose that there exist $F \in \mathfrak{I}_{2}$ and $x_{0} \in X$ such that (4.5) and the following hold:

$$
\begin{aligned}
& F(f x, g y)>\inf \{F(f z, s z), F(g z, t z)\left.: z \in G^{*} x \bigcup G^{*} y\right\}, \\
& \forall x, y \in X \text { with } f x \neq g y .
\end{aligned}
$$

Then $f$ and $s$ or $g$ and $t$ have a coincidence point in $X$.

Proof. Define $A=G^{*} x_{0}$ and $B=\bigcap_{h \in G^{*}} h \bar{A}$. As in the proof of Theorem 4.1, we infer that $B$ is compact, $h B=B \neq \varnothing$ for all $h \in G^{*}$, and there are $a, b \in B$ such that

$$
F(f a, s a)=\inf \{F(f x, s x): x \in B\}, \quad F(g b, t b)=\inf \{F(g x, t x): x \in B\} .
$$

Without loss of generality, we assume that

$$
F(f a, s a) \leq F(g b, t b) .
$$

Since $f, g, s$, and $t \in G^{*}$, it follows that $f B=g B=s B=t B=B$. Thus there exist $v, w \in B$ with $a=g v$ and $s a=g w$. We claim that $f a=s a$. If not, then $f g v \neq g w$. By virtue of (4.15), (4.16), and (4.17), we have

$$
\begin{aligned}
F(f a, s a) & =F(f g v, g w) \\
& >\inf \left\{F(f z, s z), F(g z, t z): z \in G^{*} g v \bigcup G^{*} y\right\} \\
& \geq \inf \{F(f z, s z), F(g z, t z): z \in B\} \\
& =F(f a, s a),
\end{aligned}
$$

which is a contradiction. Hence $f a=s a$. This completes the proof.

THEOREM 4.9. Let $f$ and $g$ be continuous and nearly densifying mappings from a complete metric space $(X, d)$ into itself and $G=\{f, g\}$. Suppose that there exist $F \in \mathfrak{J}_{2}$ and $x_{0} \in X$ satisfying (4.5) and

$$
\begin{array}{r}
F(f x, g y)>\inf \left\{F(f z, z), F(g z, z), F(h x, h y): z \in G^{*} x \bigcup G^{*} y, h \in C_{f} \bigcap C_{g} \bigcap G^{*}\right\}, \\
\forall x, y \in X \text { with } f x \neq g y .
\end{array}
$$

Then $f$ or $g$ has a fixed point in $X$.

Proof. It may be completed following the proof of Theorem 4.8.

\section{REFERENCES}

[1] H. Chatterjee, Remarks on some theorems of K. Iseki, Indian J. Pure Appl. Math. 10 (1979), no. 2, 158-160. MR 80d:54053. Zbl 403.54040. 
[2] M. L. Diviccaro, M. S. Khan, and S. Sessa, Common fixed point theorems for densifying mappings, Rad. Mat. 6 (1990), no. 2, 295-301. MR 91k:54074. Zbl 744.54015.

[3] B. Fisher and M. S. Khan, Results on fixed points of densifying mappings, Math. Sem. Notes Kobe Univ. 7 (1979), no. 3, 509-514. MR 81c:54062. Zbl 431.54026.

[4] M. Furi and A. Vignoli, A fixed point theorem in complete metric spaces, Boll. Un. Mat. Ital. (4) 2 (1969), 505-509. MR 41\#1034. Zbl 183.51404.

[5] Y.-Y. Huang, T.-J. Huang, and J.-C. Jeng, On common fixed points of semigroups in compact metric spaces, Indian J. Pure Appl. Math. 27 (1996), no. 11, 1073-1076. MR 97i:54051. Zbl 865.47043.

[6] K. Iséki, Fixed point theorems for densifying mappings, Math. Sem. Notes Kobe Univ. 2 (1974), no. 1, paper no. XIII, 6 pp. MR 54\#6114. Zbl 289.54031.

[7] _ Fixed point theorems for densifying mappings, Nanta Math. 9 (1976), no. 1, 50-53. MR 55\#11229. Zbl 351.54039.

[8] R. K. Jain and R. Jain, A result on fixed point of three densifying mappings, Bull. Calcutta Math. Soc. 85 (1993), no. 1, 51-54. MR 94b:54110. Zbl 782.54038.

[9] L. Janos, H. M. Ko, and K. K. Tan, Edelstein's contractivity and attractors, Proc. Amer. Math. Soc. 76 (1979), no. 2, 339-344. MR 80k:54084. Zbl 411.54031.

[10] M. S. Khan, Some fixed point theorems in metric and Banach space, Indian J. Pure Appl. Math. 11 (1980), no. 4, 413-421. MR 81c:54065. Zbl 451.54035.

[11] M. S. Khan and B. Fisher, On fixed points of densifying mappings, Math. Sem. Notes Kobe Univ. 6 (1978), no. 2, 345-349. MR 80a:54085. Zbl 404.54040.

[12] M. S. Khan and Z. Q. Liu, On coincidence points of densifying mappings, Turkish J. Math. 21 (1997), no. 3, 269-276. CMP 1482 785. Zbl 882.54036.

[13] M. S. Khan and K. P. R. Rao, A coincidence point theorem for densifying mappings, Publ. Math. Debrecen 45 (1994), no. 3-4, 365-370. MR 95k:54073. Zbl 827.54029.

[14] S. Khan, Fixed point theorem for densifying mapping, J. Indian Acad. Math. 12 (1990), no. 2, 153-155. CMP 1119 411. Zbl 757.54020.

[15] C. Kuratowski, Topologie. Vol. I, 3éme ed., Monografie Matematyczne, Tom XX, Polskie Towarzystwo Matematyczne, Warsawa, 1952 (French). MR 14,1000i. Zbl 049.39703.

[16] Z. Liu, Densifying mappings and common fixed points, Indian J. Math. 39 (1997), no. 3, 235-240. CMP 1632 868. Zbl 910.54036.

[17] Z. Q. Liu, Fixed point theorems for condensing and compact maps, Kobe J. Math. 11 (1994), no. 1, 129-135. MR 96d:54043. Zbl 857.54047.

[18]__ Fixed point theorems for densifying maps, Indian J. Math. 36 (1994), no. 2, 147150. CMP 1345 266. Zbl 859.54036.

[19] _ On densifying maps of complete metric spaces, Chinese J. Math. 22 (1994), no. 1, 47-51. MR 94k:54077. Zbl 791.54061.

[20] _ Families of mappings and fixed points, Publ. Math. Debrecen 47 (1995), no. 1-2, 161-166. CMP 1362 279. Zbl 854.54039.

[21] _ Fixed points for densifying mappings, Pure Appl. Math. Sci. 43 (1996), no. 1-2, 81-83. CMP 1627 425. Zbl 879.54052.

[22] J. Madhusudana Rao, On some theorems of Iseki, Indian J. Pure Appl. Math. 12 (1981), no. 5, 580-584. MR 82k:54073. Zbl 462.54032.

[23] R. D. Nussbaum, Some asymptotic fixed point theorems, Trans. Amer. Math. Soc. 171 (1972), 349-375. MR 46\#9817. Zbl 256.47040.

[24] R. K. Pande, A fixed point theorem for densifying mapping in complete metric space, Indian J. Pure Appl. Math. 21 (1990), no. 4, 347-349. MR 91a:54060. Zbl 703.54030.

[25]__ A fixed point theorem in bounded complete metric space, Pure Appl. Math. Sci. 31 (1990), no. 1-2, 163-165. MR 91f:54024. Zbl 712.54032.

[26] B. K. Ray and B. Fisher, Some results on fixed points for densifying mappings, Indian J. Math. 23 (1981), no. 1-3, 223-228. MR 84i:54054. Zbl 509.54043.

[27] K. P. R. Sastry and S. V. R. Naidu, Fixed point theorems for nearly densifying maps, Nepali Math. Sci. Rep. 7 (1982), no. 1, 41-44. MR 85e:54055. Zbl 551.54028. 
[28] B. S. Sharma, A fixed point theorem for densifying mappings, Pure Appl. Math. Sci. 32 (1990), no. 1-2, 49-52. CMP 1087 023. Zbl 714.54044.

[29] B. S. Sharma and S. K. Srivastava, Fixed point theorem for densifying mappings in complete metric space, Indian J. Pure Appl. Math. 22 (1991), no. 1, 5-7. MR 92b:54087. Zbl 735.54032.

Zeqing Liu: Department Of Mathematics, Liaoning Normal University, Dalian, LIAONING, 116029, CHINA

JeOng Sheok Ume: Department Of Applied Mathematics, Changwon National UniverSITY, CHANGWON, 641-773, KOREA 


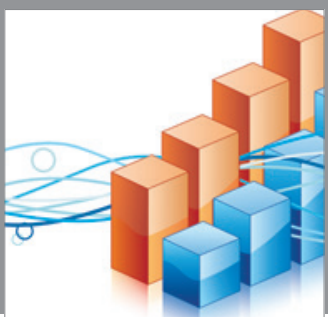

Advances in

Operations Research

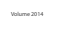

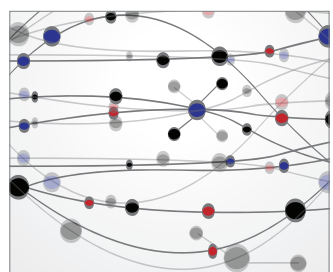

\section{The Scientific} World Journal
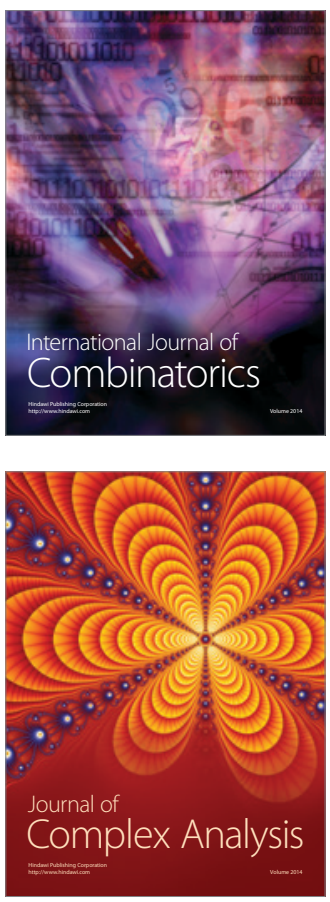

International Journal of

Mathematics and

Mathematical

Sciences
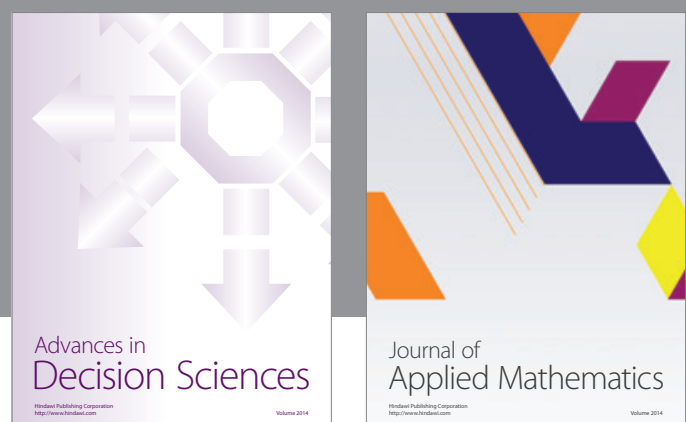

Journal of

Applied Mathematics
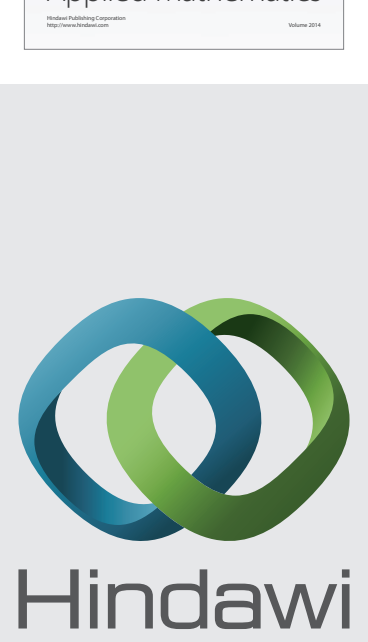

Submit your manuscripts at http://www.hindawi.com
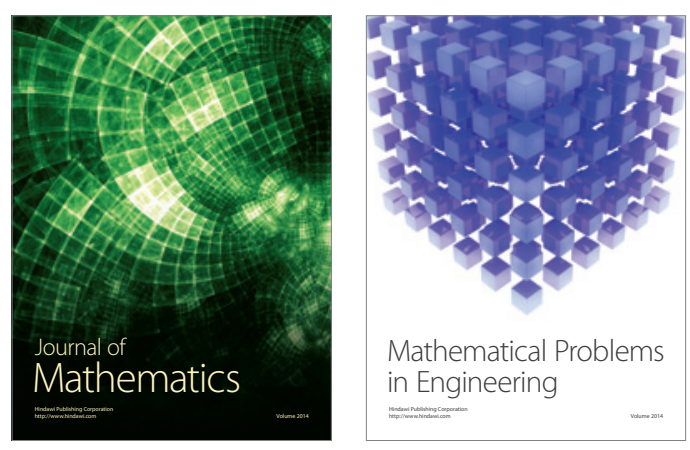

Mathematical Problems in Engineering
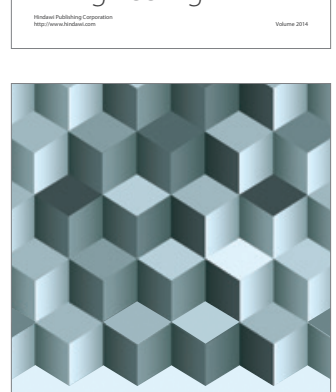

Journal of

Function Spaces
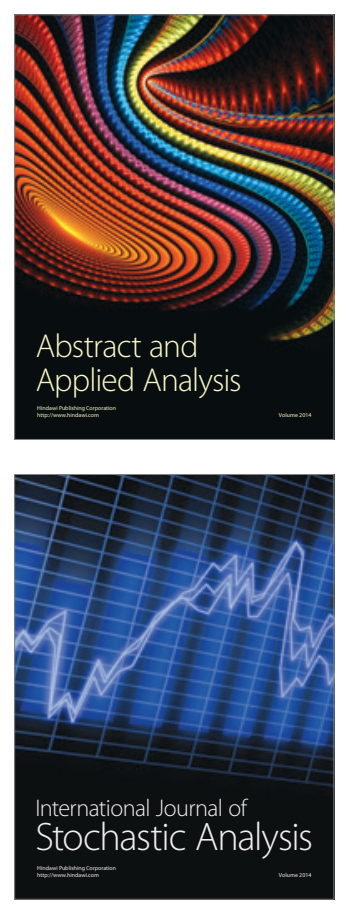

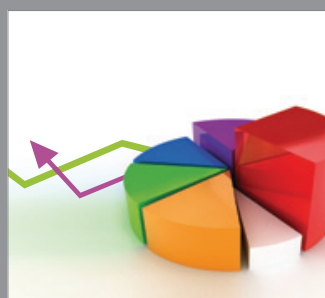

ournal of

Probability and Statistics

Promensencen
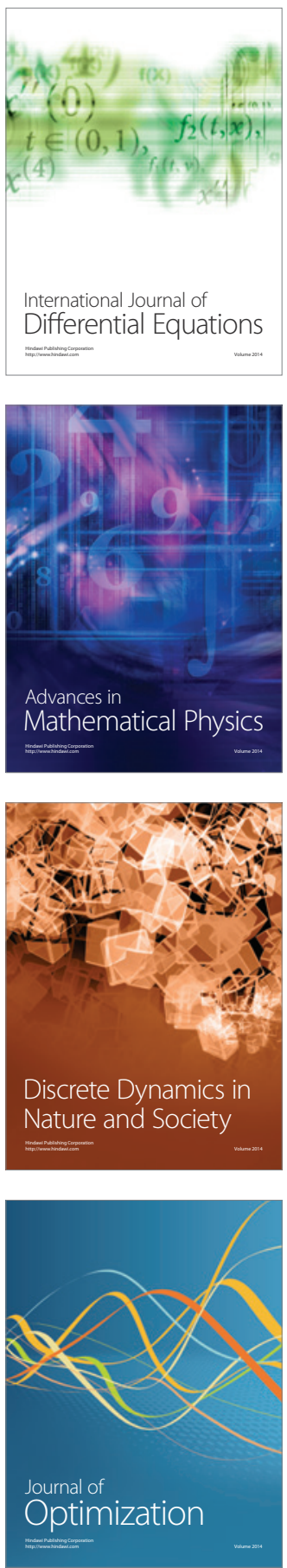